\title{
Neuroprotection for Ischemic Stroke: Two Decades of Success and Failure
}

\author{
Yu Dennis Cheng, Lama Al-Khoury, and Justin A. Zivin \\ Stroke Center, University of California School of Medicine, and University of California San Diego, La Jolla, California, $92093-0624$
}

\begin{abstract}
Summary: Alteplase (rt-PA) is the first therapy successfully developed for acute stroke therapy. The success of rt-PA spurred development of new avenues for acute stroke management. For the last two decades, a great deal of attention has been paid to neuroprotective therapies. Initial preclinical studies demonstrated numerous drugs are effective for treating acute stroke in animal models; however, subsequent clinical trials have been frustrating, and none of the agents has proven effective. The various outcomes
\end{abstract}

of preclinical and clinical trials have been the subject of much discussion. In this article, we review some key neuroprotective trials and the possible reasons for their failures. By identifying the discrepancies between preclinical studies and clinical trials, we may be able to set guidelines for future effective trials. Key Words: Neuroprotection trials, glutamate antagonists, antiinflammatory agents, ion channel blocks, free radical scavengers, neurotrophic factors, neural stem cells.

\section{INTRODUCTION}

Stroke is the leading cause of adult disability and remains the third most common cause of death in industrialized nations. ${ }^{1}$ Physicians have remained helpless regarding protection and recovery of neurons from ischemic insults. However, alteplase (rt-PA) was recently approved in the United State and Europe for acute stroke therapy. The success of rt-PA has revolutionized acute stroke management and proved that stroke is a treatable disease. This fueled the interest in the development of neuroprotective therapies.

The concept of neuroprotection mainly came from the studies of the pathology and pathophysiology of ischemic brain injury. It has been well documented that abrupt deprivation of oxygen and glucose to neuronal tissues elicits a series of pathological cascades, leading to spread of neuronal death. Of the numerous pathways identified, excessive activation of glutamate receptors, accumulation of intracellular calcium cations, abnormal recruitment of inflammatory cells, excessive production of free radicals, and initiation of pathological apoptosis are believed to play critical roles in ischemic damage, especially in the penumbral zone. Thus, it is logical to

Address correspondence and reprint requests to Justin A. Zivin, M.D., Ph.D., Department of Neurosciences, University of California San Diego, 9500 Gilman Drive, La Jolla, CA 92093-0624. E-mail: jzivin@ucsd.edu. suggest that if one is able to interrupt the propagation of these cascades, at least part of the brain tissue can be protected.

For the last two decades, neuroprotective agents designed to block these cascades (Table 1) have been investigated in animal models of cerebral ischemia. Numerous agents have been found to reduce infarct size in rodent, rabbit, and primate stroke models. ${ }^{2-5}$ However, translation of neuroprotective benefits from the laboratory bench to the emergency room has not been successful. According to a recent review, ${ }^{6}$ of 178 controlled clinical trials that were published in the stroke-related literature, more than 100 were related to neuroprotection. Reasons for the failures have led to intense discussion for the last several years. ${ }^{7-10}$ In this review, we will discuss some of the key trials of neuroprotective therapy, potential problems leading to the failures of these trials, and possible correction of those mistakes, which may form a basis for future trials.

\section{NEUROPROTECTIVE TRIALS: FAILURES BUT WORTH MENTIONING}

\section{Glutamate antagonists}

Some of the most studied neuroprotective agents are glutamate antagonists. Glutamate is the most common excitatory neurotransmitter in the CNS and is released excessively during ischemia. ${ }^{11}$ Various postsynaptic 
TABLE 1. Families of Neuroprotective Agents and Their Prototype Drugs

\begin{tabular}{lll}
\hline Proposed Mechanism of Neuroprotection & \multicolumn{1}{c}{ Drugs } & Clinical Trials and Results \\
\hline $\begin{array}{l}\text { Glutamate receptor antagonists } \\
\text { NMDA antagonists }\end{array}$ & Selfotel (CGS19755) & Complete/no benefit \\
& Eliprodil (Cerestat, CNS1102) & Halted/no benefit \\
& Aptiganel (Complete/no benefit \\
& MgSO4 & Ongoing/result pending \\
AMPA antagonist & YM872 & Ongoing/result pending \\
Ion channel modulators & & Complete/no benefit \\
Calcium channel blockers & Nimodipine & Complete/no benefit \\
Sodium channel blockers & Flunarizine & Complete/no benefit \\
Potassium channel activator & Fosphenytoin & Complete/no benefit \\
Anti-inflammatory agents & Maxipost (BMS-204352) & Complete/worsening \\
& Enlimomab & Halted/no benefit \\
Free radical scavengers & LeukArrest (Hu23F2G) & Halted/no benefit \\
& rNIF & Complete/no benefit \\
& Tirilazad (U70046F) & Complete/no benefit \\
& Citicoline (cytidyl diphosphocholine) & Ongoing/result pending \\
& Ebselen & Ongoing/result pending \\
\hline
\end{tabular}

neuron receptors are activated by glutamate including NMDA, AMPA, KA, and metabotropic receptors. Activation of most of these receptors is associated with calcium ion $\left(\mathrm{Ca}^{2+}\right)$ influx with secondary $\mathrm{Ca}^{2+}$-mediated cellular enzymatic activation leading to cell damage and cell death including necrosis and apoptosis.

Accordingly, glutamate receptor antagonists were tested in numerous preclinical and clinical trials. Three agents were tested in well-controlled trials with negative results. Selfotel ${ }^{12}$ is a potent competitive glutamate receptor antagonist but acts on various sites of the glutamate receptor complex. Eliprodil ${ }^{13-15}$ is a strong antagonist that acts on the polyamine modulator site. Aptiganel (Cerestat, CNS 1102) ${ }^{16,17}$ is a non-competitive NMDA ion channel blocker with a relatively short halflife. Phase III trials with eliprodil showed no efficacy on futility analysis, and accordingly, the trial was stopped. The phase III trial with selfotel was terminated because of an increase in the adverse event to benefit ratio. A phase III trial of aptiganel again found it ineffective for acute stroke therapy.

Despite their psychiatric and cardiovascular side effects, a role for glutamate receptor antagonists in neuroprotection may still exist. Less toxic drugs have been tested as possible glutamate antagonists in stroke. Magnesium is a calcium channel blocker and specifically antagonizes NMDA receptor-mediated calcium influx. ${ }^{18}$ The potential neuroprotective role effect of magnesium is being studied in two ongoing phase III clinical trials. In one trial, $\mathrm{MgSO}_{4}$ is administered within $2 \mathrm{~h}$ of ischemic stroke onset, ${ }^{19}$ and the other requires administering intravenous magnesium sulfate within $12 \mathrm{~h}$ after isch- emic stroke onset (the Intravenous Magnesium Efficacy in Stroke trial).

YM872, an AMPA (alpha-amino-3-hydroxy-5-methylisoxazole-4-propionate) receptor antagonist has been proven to be neuroprotective in a rat middle cerebral artery (MCA) occlusion model, ${ }^{3}$ and an ongoing phase III trial in North America is assessing its potential efficacy in conjunction with t-PA thrombolysis. No trials with metabotropic glutamate receptor agonists/antagonists have been conducted.

\section{Anti-inflammatory agents}

Neuroprotection may be achieved by targeting the inflammatory processes and mediators that contribute to the production of brain injury following ischemic stroke. Enlimomab, a murine anti-intracellular adhesion molecule (ICAM)-antibody specific to the human receptor ICAM-1 receptor, was studied in preclinical and clinical trials. By blocking the ICAM-1 receptor, and thus inhibiting neutrophil adhesion and migration through the vascular endothelium, Enlimomab was shown to reduce the damage in stroke models. $^{20,21}$ However, the clinical trial failed; patients who received Enlimomab were harmed relative to placebo controls. Another agent, LeukArrest (Hu23F2G), is a monoclonal antibody that targets the neutrophil CD11/ CD18 cell adhesion molecule. A phase III trial of LeukArrest in patients who had an ischemic stroke was terminated because the interim results were unfavorable (see $\mathrm{Hu} 23 \mathrm{~F} 2 \mathrm{G}$ phase III stroke trial, in the Internet Stroke Center Stroke Trials Directory, http://www.strokecenter.org/trials/).

Recombinant neutrophil inhibitory factor (rNIF) is a hookworm-derived agent. It reduces the inflammatory 
cascade by inhibiting the polymorphonuclear leukocyte (PMN) adhesion to the endothelium. It was established to be neuroprotective in a stroke model in rats following MCA occlusion. ${ }^{22}$ Recently, a European phase II trial studied this agent using an Bayesian Adaptive Design method, in which the agent is administered in a range of doses. An estimate of the dose-response curve is performed to deduce the most effective dosing regimen. The trial was terminated in 2003 and the results are still pending (J.A. Zivin, personal communication).

\section{Ion channel modulators}

Calcium channel blockers. Activation of intracellular destructive enzymes results from abnormal calcium influx during ischemic-triggered cascades, which leads to brain tissue damage. ${ }^{23}$ Therefore, calcium channel blockers might potentially have a neuroprotective role. Nimodipine is a dihydropyridine calcium channel blocker; it dilates the intracranial circulation and might be beneficial if it improves the regional blood flow in the margins of the brain infarct. ${ }^{5}$ This drug was studied using multiple doses in 1,064 patients treated within $48 \mathrm{~h}$ from stroke onset. No overall benefit was found, ${ }^{24}$ although a meta-analysis showed a statistically significant effect of one of the doses when the drug was administered within 12 h. ${ }^{25}$ Very Early Nimodipine Use in Stroke was another clinical trial that used nimodipine within $6 \mathrm{~h}$ after stroke onset. ${ }^{26}$ The trial was ended prematurely because of lack of benefit on a futility analysis. Moreover, upon reviewing 47 trials that included 7,665 patients and that used calcium antagonists for stroke therapy, Horn et al. ${ }^{26}$ found no benefit of these agents in stroke therapy.

Sodium channel blockers. The anti-convulsants phenytoin and fosphenytoin (a disodium phosphate ester of 3-hydroxymethyl-5,5-diphenylhydantoin) have been shown to decrease the amplitude of sodium-dependent action potentials ${ }^{27}$ and to block voltage-dependent calcium entry into synaptosomes. ${ }^{28}$ Fosphenytoin has better aqueous solubility and less irritant properties than intravenous phenytoin and is rapidly converted to phenytoin after intravenous administration. ${ }^{29}$ Fosphenytoin was studied in a phase III trial for stroke to establish whether it has any neuroprotective effects but the trial was terminated because interim analysis failed to show a benefit.

Potassium channel activator. Preclinical studies in animal stroke models showed a neuroprotective role for Maxipost (BMS-204352; Bristol-Myers Squibb, New York, NY), an activator of neuronal potassium channels. Its neuroprotective effect is produced by inducing neuronal hyperpolarization and decreasing the release of excitatory amino acids. ${ }^{30}$ A clinical phase III trial was terminated because of failure to show benefit.

\section{Free-radical scavengers}

As mentioned above, during the ischemic cascade, there is production of highly reactive oxygen free radicals due to reducing conditions, including excessive excitation of excitatory amino acid receptors. ${ }^{31}$ Oxygen free radicals beget oxygen free radicals in a vicious cycle that causes destruction of cellular membranes. Accordingly, the free oxygen radical scavengers might have a potential neuroprotective role. Preclinical studies using those agents showed effectiveness in reducing neurological damage in some animal models. ${ }^{32}$ Clinical trials have tested tirilazad (U70046F) in patients with ischemic stroke, subarachnoid hemorrhage, and head injury. ${ }^{33}$ A phase III acute stroke study was terminated prematurely because of the lack of significant benefit on an interim analysis, and another similar clinical trial was also negative. ${ }^{34}$

Citicoline (cytidyl diphosphocholine) was studied in preclinical and clinical trials. It is a phosphatidylcholine precursor that has membrane stabilization properties, but it also might have other neuroprotectant mechanisms. Preclinical studies in animal stroke models showed that citicoline improved neurological outcome and infarct size. ${ }^{35,36}$ However, the clinical phase III trials with varying dosing schedules did not show efficacy. ${ }^{37}$

NXY-059 is a nitrone-based free-radical-trapping agent which has been proven to reduce the infarct size in the transient and permanent MCA occlusion rat stroke models when administered up to $8 \mathrm{~h}$ after onset of ischemia. ${ }^{38}$ It was also shown to improve the neurologic outcome (measured by performance scores) 3 weeks and 10 weeks after the stroke, and to reduce the infarct size in a marmoset stroke model when administration was delayed for up to $4 \mathrm{~h}$ after stroke onset. ${ }^{39}$ Improvement in neurologic function has also been established in a rabbit embolic stroke model ${ }^{40}$ as well as in a hemorrhagic rat stroke model. ${ }^{41}$ A safety trial tested NXY-059 in stroke patients and this agent was found to be safe in doses that were neuroprotective in the animal models. ${ }^{42}$ Accordingly, a clinical trial phase IIb/III has been designed and started. NXY-059 is to be given within $6 \mathrm{~h}$ from stroke onset, and in combination with t-PA.

\section{$\boldsymbol{\gamma}$-Aminobutyric acid receptor antagonists}

Clomethiazole is a $\gamma$-aminobutyric acid (GABA) receptor antagonist derived from the thiazole moiety of thiamine. It has been used as an anticonvulsant, sedative, and hypnotic agent and for the treatment of alcohol withdrawal symptoms. ${ }^{43}$ Clomethiazole interacts with the GABA receptor and potentiates the effect of the neurotransmitter GABA. This agent has neuroprotectant actions in both global and focal ischemia models, as shown by various outcome measures such as histopathology, excitatory amino acid release in vivo, and edema formation. ${ }^{44}$ However, a phase III trial that included 1,360 patients failed to show benefit except for the subgroup of 
total anterior circulation strokes (TACS) in which it may have shown some benefit. ${ }^{45}$ Diazepam, a GABA antagonist, is being investigated for a potential neuroprotective effects in acute stroke in a multi-centered phase III European trial with initiation of treatment within $12 \mathrm{~h}$ from onset.

\section{Serotonin agonists}

Serotonin agonists may serve as neuroprotectants by activating postsynaptic serotonin receptors $\left(5-\mathrm{HT}_{1 \mathrm{~A}}\right)$ with secondary increase in potassium efflux, inhibition of cell excitability during the ischemic insult, and protection of neurons from glutamate-mediated neuronal death. Repinotan (BAY X3702) is one of these agonists that has a high affinity for serotonin $\left(5-\mathrm{HT}_{1 \mathrm{~A}}\right)$ receptors. It has been shown to reduce excitotoxic neuronal death and was shown to be an effective neuroprotectant in a rat focal ischemic brain injury. ${ }^{46} \mathrm{~A}$ phase II clinical trial studied Repinotan in 240 patients and showed a better neurologic and functional outcome at 4 weeks and 3 months with a dose of $1.25 \mathrm{mg} /$ day for 3 days when the agent was given within $6 \mathrm{~h}$ after stroke onset. A phase IIb trial has been projected to include 680 patients with a window of administration of $4.5 \mathrm{~h}$.

\section{Caspase inhibitors}

Apoptosis has been suggested to be one of the key elements in delayed brain injury after ischemic stroke. One of the important factors in the apoptosis cascade is caspase activation. Caspases are a group of cysteine proteases that cleave various proteins associated with neuronal apoptosis including poly(ADP-ribose) polymerase (PARP), DNA-dependent protein kinase, U1-soluble nuclear RNA polymerase (U1-snRNP), spectrin, lamin A, actin, and protein kinase C. Activation of caspases has been shown in some animal ischemic models, while inhibition of caspase activity reduced infarction size in rodent ischemia stroke models. ${ }^{47,48}$ Chen et al ${ }^{49}$ further demonstrated that caspase inhibitor Z-VAD improved the survival of grafted bone marrow cells in rats subjected to unilateral MCA occlusion, and significantly improved their functional outcome. Although evidence in preclinical studies is reasonable, none of the caspase inhibitors has yet been tested in a clinical trial, probably because many of the caspase inhibitors are irreversible and have poor brain penetration.

\section{Other potential neuroprotective agents}

A variety of other drugs that have potential neuroprotective properties have also been tested in clinical trials. Nalmefene (Cervene) is an opioid antagonist with relative selectivity for kappa opiate receptors. It has been shown to reduce neuronal ischemic injury by inhibiting glutamate release. ${ }^{50,51}$ However, a phase III trial reported no significant difference in 3-month functional outcome compared with placebo. ${ }^{52}$ Lubeluzole is a drug that was shown to inhibit nitric oxide production and has a number of other actions. A series of phase I to III trials were conducted to examine its therapeutic efficacy for ischemic stroke. A recent phase III trial failed to show significant benefits compared to placebo (J.A. Zivin, personal communication).

Gangliosides are a family of large-molecular-weight glycoshingolipids. Preclinical studies indicated that gangliosides may protect neurons by multi-factorial mechanisms, which include normalization of altered protein phosphorylation, increasing brain-derived neurotrophic factor (BDNF) expression, blocking overstimulation of excitatory amino acid, and stabilization of plasma membrane structure. A number of experiments in ischemic brain injury models have shown protection when the ganglioside GM1 was administered..$^{53,54}$ While preclinical evidence was moderately convincing, the Italian Acute Stroke Study was disappointing. The trial enrolled 502 patients within $12 \mathrm{~h}$ after stroke onset with the drug continuously administered for 15 days. It failed to show any beneficial effects at the 3-month endpoint assessment. ${ }^{55}$

\section{Neurotrophic factors}

Neurotrophic factors are a group of proteins that are involved in cell proliferation, migration, differentiation and development of the nervous system. In the adult $\mathrm{CNS}$, neurotrophic factors are thought to play roles in normal maintenance and survival of neuronal cells by keeping cell survival genes activated and suicide genes suppressed. ${ }^{56}$ For this reason, deprivation of neurotrophic factors in the stroke penumbra may activate neuronal apoptosis and lead to cell death. In preclinical studies, neurotrophic factors such as nerve growth factor, BDNF, ciliary neurotrophic factor, glial-derived neurotrophic factor, vascular endothelial growth factor, and insulin-like growth factor (IGF)-1 have all been shown to reduce infarct size in animal models, both in adult ${ }^{57-59}$ and immature animals. ${ }^{60,61}$ However, only basic fibroblast growth factor (bFGF) progressed to a clinical trial. bFGF is a polypeptide of 154 amino acids, which is present at high levels in brain extracts. It increases neuronal survival, has trophic effects on brain glial and endothelial cells and is a potent systemic and cerebral vasodilator. ${ }^{62,63}$ It has been demonstrated that administration of bFGF after permanent ${ }^{64}$ or transient MCA occlusion ${ }^{65}$ significantly reduced infarct volumes. Although a phase I trial in stroke patients was promising, the U.S. phase II trial was halted because patients that received bFGF did worse compared to the placebo group, and the companion European trial failed to show efficacy.

\section{Neural stem cells}

Neural stem cells are able to differentiate and regenerate in response to both internal and external stimuli. They are mainly found in developing brain; however, neural stem cells were recently found concentrated in the 
subgranular zone of the hippocampal dentate gyrus or the subventricular zone, ${ }^{66}$ as well as in other brain regions including the frontal cortex of adult brains. ${ }^{67}$ These discoveries suggest that, in certain circumstances, neural stem cells may be able to regenerate and restore loss of brain function in injuries such as stroke. In fact, regeneration of neurons has been observed in response to ischemic insults; Takagi et $\mathrm{al}^{68}$ demonstrated an increase in proliferation of neuronal precursor cells in the hippocampal dentate gyrus in a mouse transient ischemic model. Nakatomi et al. ${ }^{69}$ showed cell proliferation in response to ischemia and subsequent migration into the hippocampus to regenerate new neurons. Intraventricular infusion of growth factors markedly augments these responses, thereby increasing the number of newborn neurons. Furthermore, cell proliferation and differentiation from ependymal, subependymal, and choroid plexus cells in response to stroke were also demonstrated. ${ }^{70}$ However, the therapeutic mechanisms of stem cells have been controversial. While many researchers expected neural stem cells grafted into infarct sites to restore function by "filling in the hole," Modo et al. ${ }^{71}$ demonstrated that implanted stem cells improved behavioral functions of MCA occluded rats without change of infarction size. More interestingly, using bone marrow stromal cells, which have been shown to develop into neuroectodermal cells including neurons, ${ }^{72} \mathrm{Li}$ et $\mathrm{al}^{73}$ were able to show an enhancement of functional recovery of MCA infarcted rats even with bone marrow stromal cells were given intravenously $24 \mathrm{~h}$ after stroke. These studies strongly suggest that neural stem cells might facilitate stroke recovery by mechanisms other than simply replacing lost neurons.

Even though the therapeutic effects of neural stem cells on ischemic stroke in animal models are becoming more and more convincing, they have not yet progressed to clinical trials. However, a small phase I study was conducted by Kondziolka et al ${ }^{74}$ by using LBS-Neurons, a cell line derived from human teratocarcinoma. LBSNeurons were transplanted into the brains of 12 patients with basal ganglia stroke and fixed motor deficits. The study demonstrated that there was a mean improvement of 2.9 points in the European Stroke Scale assessed 12 to 18 months after cell implantation. No adverse effects were observed during this study period; these results need to be viewed with great caution. First, LBS-Neurons are cells derived from human teratocarcinoma, and a 12- to 18-month follow-up may not be sufficient to ensure the safety of the transplants. Second, this is a phase I study that was not powered to prove efficacy.

\section{Hypothermia}

It has been well documented that mild $\left(34^{\circ} \mathrm{C}\right)$ to moderate $\left(32^{\circ} \mathrm{C}\right)$ systemic hypothermia protects brain from some ischemic damage in various animal models. Although the exact mechanisms are unknown, a reduction of body temperature, especially brain temperature, may lead to reduced cerebral oxygen consumption, decreased intracellular lysosomal enzyme activity, suppressed free radical formation, protection of the fluidity of the cell membranes, reduced intracellular acidosis, and inhibition of cell damage mediated by excitatory neurotransmitters. ${ }^{75}$

Several clinical studies suggested that mild hypothermia improved neurologic outcome in patients after cardiac arrest. A case-control multi-center trial demonstrated that cardiac arrest patients who were treated with mild externally applied hypothermia $\left(33^{\circ} \mathrm{C}\right)$ had significantly better neurological outcome and less mortality than those who were maintained normothermic. ${ }^{75}$ Acute hyperthermia, on the other hand, has been associated with a higher mortality rate 1 year post-cardiac-arrest, as compared to normothermic patients. ${ }^{76}$

Recently, Krieger et $\mathrm{al}^{77}$ published a pilot study, investigating moderate hypothermia therapy in ischemic stroke. Nineteen patients with major acute ischemic stroke [National Institutes of Health Stroke Scale (NIHSS) $>15$ ] were randomly assigned to either hypothermic $\left(32^{\circ} \mathrm{C}\right)$ or control (maintained normothermic) groups. Patients in the hypothermic group were subjected to surface cooling with a cold blanket. The therapeutic window for hypothermic treatment was $6 \mathrm{~h}$ after stroke onset, and thrombolytic therapy was given whenever patients were eligible. Although complications such as bradycardia, ventricular ectopy, hypotension, melena, infections, and rebound hyperthermia were observed, the treatment was considered safe. ${ }^{77}$

There are two main obstacles to surface cooling hypothermia: slow induction and intense patient shivering. To overcome these problems, endovascular cooling was introduced. In general, a catheter with a closed-loop circulation system embedded was attached to a temperature controlling system. The catheter was then inserted in the inferior vena cava to cool circulating blood and regulate body temperature. Georgiadis et al. ${ }^{78}$ reported 6 patients who suffered severe acute ischemic stroke and were treated with endovascular hypothermia. During the hypothermia, the minimal temperature achieved was $32.2^{\circ} \mathrm{C}$. According to their study, the procedures were safe, and the adverse effects were similar to those encountered in surface cooling. To further evaluate its safety and efficacy, several clinical trials are currently being conducted.

\section{FROM BENCH-TOP TO BEDSIDE: POTENTIAL PROBLEMS AND POSSIBLE SOLUTIONS}

Although evidence from preclinical studies has been exciting and many drugs have progressed to multi-center clinical trials, none of the neuroprotective agents has been proven to be clinically beneficial. In this section, 
potential problems that hindered the translation of preclinical success into clinical practice will be discussed. By learning from our past mistakes, we may be able to have more successful studies in the future.

\section{Problems that may have caused the failure of past clinical trials}

The discrepancies between preclinical studies and clinical trials may be the cause of some of the problems encountered previously. Below are five suggested discrepancies that may explain some of the disappointing outcomes.

Discrepancies on the outcome measures. In most preclinical studies, efficacy of neuroprotective agents was detected by reduction of histological infarction volume. However, in clinical trials, neuroprotective efficacy is measured by neurological function such as the NIH Stroke Scale and the modified Rankin Scale. Infarction volumes correlate poorly with functional outcome because small lesions in critical locations can produce major functional deficits. Conversely, large lesions in relatively silent areas cause little detectable function loss. While most of animal stroke models used in preclinical neuroprotection studies are MCA occlusion models, patients enrolled into clinical trials often include infarcts of diverse brain regions. ${ }^{6}$ Thus, some animal models may be poor predictors of clinical trial results.

Another factor that may play a role in the discrepancies between preclinical and clinical study outcomes is the difference in the composition of brain between rodents and humans. It has been reported that more than $90 \%$ of brain tissue in rodents is composed of gray matter, whereas in human, gray matter makes up about $50 \%$ of the brain. ${ }^{79}$ Even in the most homogeneous population of cortical stroke patients, the damage to white matter in humans will be significantly larger than in rodent models. This is not necessarily important, but is a reason to be cautious in extrapolation of rodent model results to humans, particularly for drugs that have differential effects in white versus gray mater.

Discrepancies on functional assessment. Although in recent animal model studies of neuroprotective agents, more attention has been paid to functional assessment, the tests chosen in preclinical studies may not reflect those used in clinical trials. Numerous functional measures have been developed for animal studies, including limb pacing, beam and grid walking, grip strength, Tmaze retention tests, Morris water maze results, and radial arm maze performance. ${ }^{80}$ Many researchers chose the tests according to their own expertise, and not necessarily because the tests represent important aspects of functional outcomes in animals.

In clinical trials, measures of impairment, disability, and handicap have all been used. The NIH Stroke Scale, modified Rankin Scale, and Barthel Index are the most popular endpoint measures for clinical trials. ${ }^{81}$ The NIH Stroke Scale mainly measures neurological impairment, the modified Rankin Scale assesses global change in activity and lifestyle, and Barthel Index focuses on the impact of the stroke on patients' activities in daily life. However, none of these clinical scales have been proven to correlate well with infarct volumes or any particular battery of preclinical function tests.

Discrepancies of pre-morbid conditions. Another discrepancy between laboratory animals and stroke patients is their pre-morbid conditions. In stroke models, researchers usually choose young, healthy animals. However, stroke patients are usually old and suffer from multiple chronic diseases such as arteriosclerosis, hypertension, diabetes, hyperlipidermia, and prior stroke. Comorbidities of patients can affect their functional outcome ${ }^{82}$ thus altering the measurements of drug efficacy and safety.

Discrepancies of therapeutic windows. In many animal studies, neuroprotective agents were given before or immediately after the onset of ischemia. We now understand that neuroprotective agents should show efficacy for at least 2 to $3 \mathrm{~h}$ after artery occlusion ${ }^{83,84}$; However, many pharmaceutical companies view such a short therapeutic windows as a major obstacle to marketing their drugs, so most clinical trials have chosen a window of 6 or more hours after stroke onset. Some trials even extended the therapeutic window to as long as 12 days. $^{6}$ Although an animal study indicated that neuronal death was observed as late as 14 days after artery occlusion, ${ }^{85}$ the role of this type of delayed neuronal death in human stroke is yet to be determined. It is probable that the lengthy therapeutic windows used in most clinical trials are simply too long for any meaningful neuroprotection to be shown.

Discrepancies of drug-dosing schedules. Another key disagreement between animal studies and clinical trials is drug-dosing regimens. To avoid toxicity, some trials have used a dose that was significantly lower than that shown in animal studies to be efficacious. ${ }^{86}$ On the other hand, while in most animal studies drugs were administered for only short periods before or after the ischemic insult, the dosing schedules in clinical trials have been highly variable, ranging from a single intravenous infusion to multiple oral doses for up to 3 months. ${ }^{87}$ While a single dose may not provide enough protection to last beyond the propagation of post-ischemic injury, prolonged administration may be excessive and produce deleterious side effects or reduced therapeutic efficacy if the dose-response characteristics of the drug are an inverted $U$-shape.

\section{Possible answers for future clinical trials}

Final dose selection should be based on preclinical studies and phase I and II trials. It is obvious that 
drugs chosen for neuroprotective trials should have reliable preclinical studies; however, the doses that were shown to be effective in animals may not always be appropriate for stroke patients. To identify the most appropriate dose, Bayesian techniques have recently been employed in advanced drug trials. ${ }^{88}$ Patients enrolled in the trial are randomly assigned to a range of doses initially, based on the preclinical studies and previous phase I trials. The outcome data from each patient is used iteratively by a computer that is programmed with a pre-specified decision rule. As the trial proceeds, the optimal dose will be generated. Bayesian techniques allow statisticians to eliminate ineffective or potentially harmful doses, and may reduce total sample size, particularly in a subsequent phase III trial.

Time window for initiation of drugs must reflect the preclinical studies and phase I and II trials. To maximize possible therapeutic benefits, the first dose of neuroprotective treatment should be given as soon as possible after stroke onset. If the drug is proven to be beneficial, follow-up trials with an extended therapeutic window may be attempted. Even though a long timewindow may be selected, clinical trialists should be encouraged to enroll patients as soon as possible after symptom onset, and there should often be stratification by enrollment time.

Patient selection should reflect the therapeutic target. In different parts of the brain, the mechanism of injury elicited by ischemic stroke may vary because of differing cell receptor types. For instance, if a drug is mainly targeting gray matter, patients with subcortical lacunar infarcts that are mainly confined to the white matter should be excluded; computed tomography scans and various magnetic resonance imagining techniques may be useful tools, but this has not yet been proven. Furthermore, the severity of stroke may also affect the study outcome. While patients with mild strokes (NIHSS $<6)$ may have a better chance for spontaneous recovery, patients with severe strokes (NIHSS $>22$ ) are very unlikely to have full recovery. For example, trials should enroll only patients with NIHSS scores between 7 and 22 to have a reasonable chance of showing therapeutic benefit, if any is present.

Endpoint and outcome measurement should be appropriate. In most previous acute stroke trials, 90 days was chosen for primary endpoint assessment. Although this may be appropriate for most studies, an adjustment of endpoint assessment time may be needed in some instances. Medical complications unrelated to tested drugs may alter the outcome with prolonged follow-up. An earlier endpoint may help to eliminate those unwanted effects. Drugs thought to have restorative effects might need longer follow-up periods to allow beneficial effects to be shown.

The outcome measures chosen is another critical issue.
Although measures of impairment, disability, and handicap have all been used, none was proven to be superior to the others; therefore, the simplest (the modified Rankin Scale) is probably satisfactory. Although the modified Rankin Scale has been criticized for its subjectivity, when administered with structured interview it was shown to have reduced the variability and bias between raters. ${ }^{89}$ On the other hand, the global statistic approach with multiple predefined outcome measures was employed in NINDS t-PA stroke trials. ${ }^{90}$ It was slightly more effective than any single outcome measure. It is possible that, in the future, imaging outcome measures may be included in the global statistic to increase its power.

Combination therapy trials. Thrombolytic therapy with rt-PA has become the standard of care in the U.S. and a number of other countries. Acute stroke patients who present within $3 \mathrm{~h}$ of symptom onset will be given rt-PA if they qualify, and any neuroprotective agent will have to be administered in combination with rt-PA. In fact, there is preclinical evidence indicating that combinations of rt-PA and some neuroprotective agents may result in positive interactions. ${ }^{91,92}$ Administration of rt-PA increases perfusion of ischemic penumbra, preserves salvageable core tissue, and may extend the therapeutic window for neuroprotective therapy. ${ }^{91,92} \mathrm{Co}-\mathrm{ad}-$ ministration of neuroprotective agents may extend the therapeutic window of rt-PA. ${ }^{93}$ Furthermore, reperfusion with a thrombolytic permits the neuroprotective agent to better penetrate the previously ischemic region.

Various families of neuroprotective agents target different aspects of the neurodegenerative cascade. Thus, used in combination, they may have synergistic effects against ischemic injury. For this reason, the dose of each drug may be reduced to limit the drug toxicity and increase patient tolerability; however, combining two or more drugs in the same study will add to the complexity of trial design.

\section{SUMMARY}

For the last two decades, the search for the neuroprotective therapies for acute ischemic stroke has experienced a roller coaster ride. Early success in the preclinical studies may have prematurely pushed numerous agents into clinical trials. Translating bench success to the bedside proof of efficacy and safety has been frustrating. Lack of satisfactory animal models resembling the human disease, and discrepancies between preclinical studies and clinical trials have proven costly. However, we have one important success: rt-PA is approved for acute stroke management in many countries because it is truly effective and safe when administered properly. Much more needs to be done; learning from past failures, 
we have reasons to believe that at least some of the neuroprotective agents will be proven to be beneficial.

\section{REFERENCES}

1. American Stroke Association. Heart Disease and Stroke Statistics2003 Update. Dallas: American Heart Association, 2003.

2. Marshall J, Duffin KJ, Green AR, Ridley R. NXY-059, a free radical-trapping agent, substantially lessens the functional disability resulting from cerebral ischemia in a primate species. Stroke 32:190-198, 2001.

3. Shimizu-Sasamata M, Kano T, Rogowska J, Wolf GL, Moskowitz MA, Lo EH. YM872, a highly water-soluble AMPA receptor antagonist, preserves the hemodynamic penumbra and reduces brain injury after permanent focal ischemia in rats. Stroke 29: 2141-2148, 1998.

4. Lapchak P et al. A novel thrombolytic that improves behavioral outcome after embolic strokes in rabbits. Stroke 33:2279-2284, 2002.

5. Steen P, Newberg LA, Milde JH, Michenfelder JD. Nimodipine improves cerebral blood flow and neurologic recovery after complete cerebral ischemia in the dog. J Cereb Blood Flow Metab 3:38-43, 1983.

6. Kidwell CS, Liebeskind DS, Starkman S, Saver JL. Trends in acute ischemic stroke trial through the 20th century. Stroke 32:1349$1359,2001$.

7. Zivin JA, Grotta JC. Animal stroke models: they are relevant to human disease. Stroke 21:981-983, 1990.

8. Gorelick PB. Neuroprotection in acute ischaemic stroke: a tale of for whom the bell tolls. Lancet 355:1925-1926, 2000.

9. Morgenstern LB. What have we learned from clinical neuroprotective trials? Neurology 57:S45-S47, 2001.

10. Gladstone DJ, Black SE, Hakim AM. Toward wisdom from failure: lessons from neuroprotective stroke trials and new therapeutic directions. Stroke 33:2123-2136, 2002.

11. Guyot LL, Diaz FG, O’Regan MH, Song D, Phillis JW. Real-time measurement of glutamate release from the ischemic penumbra of the rat cerebral cortex using a focal middle cerebral artery occlusion model. Neurosci Lett 299:37-40, 2001.

12. Boast CS, Gerhardt B, Pastor G, Lehmann J, Etienne PE, Liebman JM. The N-methyl-D-aspartate antagonist CGS19755 and CPP reduce ischemic brain damage in gerbils. Brain Res 442:345-348, 1988.

13. Gotti B, Duverger D, Bertin J, Carter C, Dupont R, Frost J et al. Ifenprodil and SL 82.0715 as cerebral anti-ischemic agents. 1. Evidence for efficacy in models of focal cerebral ischemia. $J$ Pharmacol Exp Ther 247:1211-1221, 1988.

14. O'Neill MJ, Hicks C, Ward M. Neuroprotective effects of 7-nitroindazole in gerbil model of global cerebral ischemia. Eur $J$ Pharmacol 310:115-122, 1996.

15. Patat A, Molinjier P, Hergueta T, Brohier S, Zieleniuk I, Danjou P et al. Lack of amnestic, psychotomimetic or impairing effect on psychomotor performance of eliprodil, a new NMDA antagonist. Int Clin Psychopharmacol 9:155-162, 1994.

16. Aronowski J, Ostrow P, Samways E, Strong R, Zivin JA, Grotta JC. Graded bioassay for demonstration of brain rescue from experimental acute ischemia in rats. Stroke 25:2235-2240, 1994.

17. Cohen RA, Hasegawa Y, Fisher M. Effects of a novel NMDA receptor antagonist on experimental stroke quantitatively assessed by spectral EEG and infarct volume. Neurol Res 16:443-448, 1994.

18. Muir KW. New experimental data on the efficacy of pharmacological magnesium infusions in cerebral infarcts [abstract]. Magnes Res 11:43-56, 1998.

19. Saver JL, Kidwell CS, FAST-MAG Trial Group. The Field Administration of Stroke Therapy-Magnesium (FAST-MAG) phase 3 trial. Paper presented at The American Heart Association 27th International Stroke Conference, Ongoing clinical trial session, 2002.

20. Clark WM, Madden KP, Rothlein R, Zivin JA. Reduction of central nervous system ischemic injury by monoclonal antibody to intercellular adhesion molecule. J Neurosurg 75:623-627, 1991.

21. Zhang RL, Chopp M, Jiang N,Tang WX, Prostak J, Manning AM et al. Anti-intercellular adhesion molecule- 1 antibody reduces isch- emic cell damage after transient but not permanent middle cerebral artery occlusion in the Wistar rat. Stroke 26:1438-1443, 1995.

22. Jiang N, Chopp M, Chahwala S. Neutrophil inhibitory factor treatment of focal cerebral ischemia in the rat. Brain Res 788:25-34, 1998.

23. Ohta K, Graf R, Rosner G, Heiss WD. Calcium ion transients in peri-infarct depolarizations may deteriorate ion homeostasis and expand infarction in focal cerebral ischemia in cats. Stroke 32:535543,2001

24. The American Nimodipine Study Group. Clinical trial of nimodipine in acute ischemic stroke. Stroke 23:3-8, 1992.

25. Mohr JP, Ogogozo JM, Harrison MJG. Meta-analysis of oral nimodipine trials in acute ischemic stroke. Cerebrovasc Dis 4:197203, 1994.

26. Horn J, de Haan R, Vermeulen M, Limburg M. Very early nimodipine use in stroke (VENUS). Stroke 32:461-465, 2001.

27. McLean MJ, MacDonald RL. Multiple action of phenytoin on mouse spinal cord neurons in cell culture. $J$ Pharmacol Exp Ther 227:779-789, 1983

28. Ferrendelli JA, Danials-McQueen S. Comparative actions of phenytoin and other antiepileptic drugs on potassium-and veratridinestimulated calcium uptake in synaptosomes. $J$ Pharmacol Exp Ther 220:29-34, 1983.

29. Bebin M, Bleck TP. New anticonvulsant drugs: focus on flunarizine, fosphenytoin, midazolam and stiripentol. Drugs 48:153-171, 1994.

30. Gribkoff VK, Starrett JE Jr, Dworetzky SI, et al. Targeting acute ischemic stroke with a calcium-sensitive opener of maxi-K potassium channels. Nat Med 7:471-477, 2001.

31. Cheng Y, Sun AY. The biomechanisms of kainate-induced neurotoxicity involved in oxidative insult. Neurochemical Res 19:15571564, 1994.

32. Lapchak PA, Chapman DF, Zivin JA. Pharmacological effects of the spin trap agents N-t-butyl-phenylnitrone (PBN) and 2,2,6,6tetramethylpiperidine-N-oxyl (TEMPO) in a rabbit thromboembolic stroke model: combination studies with the thrombolytic tissue plasminogen activator. Stroke 32:147-153, 2001.

33. Haley EC, Kassell NF, Apperson-Hansen C, Maile MH, Alves WM. A randomized, double-blind, vehicle-controlled trial of tirilazad mesylate in patients with aneurysmal subarachnoid hemorrhage: a cooperative study in North America. J Neurosurg 86:467474, 1997.

34. Haley EC Jr. High-dose tirilazad for acute stroke (RANTTAS II). RANTTAS II investigators. Stroke 29:1256-1257, 1998.

35. D'Orlando KJ, Sandage BW Jr. Citicoline (CDP-choline): mechanisms of action and effects in ischemic brain injury. Neurol Res 17:281-284, 1995

36. Schabitz W, Ali F, Irie K, Sandage BW Jr, Locke KW, Fisher M. Synergistic effects of a combination of low-dose basic fibroblast growth factor and citicoline after temporary experimental focal ischemia. Stroke 30:427-432, 1999.

37. Clark WM, Warach SJ, Pettigrew LC, Gammans RE, Sabounjian LA. A randomized dose-response trial of citicoline in acute ischemic stroke patients. Citicoline Stroke Study Group. Neurology 49:671-678, 1998

38. Zhao Z, Cheng M, Maples KR, Ma J, Buchan A. NXY-059, a novel free radical trapping compound, reduces cortical infarction after permanent focal cerebral ischemia in the rat. Brain Res 909: 46-50, 2001

39. Marshall JWB, Duffin KJ, Green AR, Ridley RM. NXY-059, a free radical-trapping agent, substantially lessens the functional disability resulting from cerebral ischemia in a primate species. Stroke 32:190-198, 2001

40. Lapchak PA, Araujo DM, Song D, Wei J, Zivin JA. Neuroprotective effects of the spin trap agent disodium-[(tert-butylimino)methyl]benzene-1,3-disulfonate N-oxide (generic NXY-059) in a rabbit small clot embolic stroke model: combination studies with the thrombolytic tissue plasminogen activator. Stroke 33:14111415, 2002.

41. Peeling J, Del Bigio MR, Corbett D, Green AR, Jackson DM. Efficacy of disodium 4-[(tert-butylimino)methyl]benzene-1,3-disulfonate $N$-oxide (NXY-059), a free radical trapping agent, in a rat 
model of hemorrhagic stroke. Neuropharmacology 40:433-439, 2001.

42. Lees KR, Sharma AK, Barer D, Ford GA, Kostulas V, Cheng YF et al. Tolerability and pharmacokinetics of the nitrone NXY-059 in patients with acute stroke. Stroke 32:675-680, 2001.

43. Evans JG, Feuerlein W, Glatt MM, Kanowski S, Scott DB. Chlomethiazole 25 years: recent developments and historical perspectives. Acta Psychiatr Scand Suppl 73[Suppl 327]:198, 1986.

44. Green AR. Clomethiazole (Zendra) in acute ischemic stroke: basic pharmacology and biochemistry and clinical efficacy. Pharmacol Ther 80:123-147, 1998.

45. Lyden P, Jacoby M, Schim J, Albers G, Mazzeo P, Ashwood T et al. The clomethiazole acute stroke study in tissue-type plasminogen activator-treated stroke (CLASS-T): final results. Neurology 57:1199-1205, 2001.

46. Alessandri B, Tsuchida E, Bullock RM. The neuroprotective effect of a new serotonin receptor agonist, BAY X3702, upon focal ischemic brain damage caused by acute subdural hematoma in the rat. Brain Res 845:232-235, 1999.

47. Cheng Y, Deshmukh M, D'Costa A, Demaro JA, Gidday JM, Shah A et al. Caspase inhibitor affords neuroprotection with delayed administration in a rat model of neonatal hypoxic-ischemic brain injury. J Clin Invest 101:1992-1999, 1998.

48. Endres M, Namura S, Shimizu-Sasamata M, Waeber C, Zhang L, Gomez-Isla $\mathrm{T}$ et al. Attenuation of delayed neuronal death after mild focal ischemia in mice by inhibition of the caspase family. J Cereb Blood Flow Metab 18:238-247, 1998.

49. Chen J, Li Y, Wang L, Lu M, Chopp M. Caspase inhibition by $\mathrm{Z}-\mathrm{VAD}$ increases the survival of grafted bone marrow cells and improves functional outcome after MCAo in rats. J Neurol Sci 199:17-24, 2002.

50. Yum SW, Faden AI. Comparison of the neuroprotective effects of the N-methyl-D-aspartate antagonist MK-801 and the opiate-receptor antagonist nalmefene in experimental spinal cord ischemia. Arch Neurol 47:277-281, 1990.

51. Graham SH, Shimizu H, Newman A, Weinstein P, Faden AI. Opioid receptor antagonist nalmefene stereospecifically inhibits glutamate release during global cerebral ischemia. Brain Res 632: 346-350, 1993.

52. Clark WM, Raps EC, Tong DC, Kelly RE. Cervene (Nalmefene) in acute ischemic stroke : final results of a phase III efficacy study. The Cervene Stroke Study Investigators. Stroke 31:1234-1239, 2000.

53. Leon A, Lipartiti M, Seren MS, Lazzaro A, Mazzari S, Koga T et al. Hypoxic-ischemic damage and the neuroprotective effects of GM1ganglioside. Stroke 21[Suppl 11]:11195-11197, 1990.

54. Carolei A, Fieschi C, Bruno R, Toffano G. Monosialoganglioside GM1 in cerebral ischemia. Cerebrovasc Brain Metab Rev 3:134157, 1991.

55. Argentino C, Sacchetti ML, Toni D, Savoini G, D'Arcangelo E, Erminio F et al. GM1 ganglioside therapy in acute ischemic stroke: Italian Acute Stroke Study-Hemodilusion + drug. Stroke 20: 1143-1149, 1989.

56. Jessell TM, Sanes JR. The generation and survival of nerve cells. In: Principles of neural science (Kandel ER, Schwartz JH, Jessell TM, eds), pp 1041-1062. New York: McGraw-Hill, 2000.

57. Zhang Y, Pardridge WM. Neuroprotection in transient focal brain ischemia after delayed intravenous administration of brain-derived neurotrophic factor conjugated to a blood-brain barrier drug targeting system. Stroke 32:1378-1384, 2001.

58. Wen TC, Matsuda S, Yoshimura T, Kawabe T, Sakanaka M. Ciliary neurotrophic factor prevent ischemic-induced learning disability and neuronal loss in gerbils. Neurosci Lett 191:55-58, 1995.

59. Abe K. Therapeutic potential of neurotrophic factors and neural stem cells against ischemic brain injury. J Cereb Blood Flow Metab 20:1393-1408, 2000.

60. Holtzman DM, Sheldon R, Cheng Y, Jaffe W, Ferriero DM. NGF protects the neonatal brain against hypoxic-ischemic injury. Ann Neurol 39:114-122, 1996.

61. Cheng Y, Gidday JM, Yan Q, Shah AR, Holtzman DM. Marked age-dependent neuroprotection by brain derived neurotrophic factor against neonatal hypoxic-ischemic brain injury. Ann Neurol 41:521-529, 1997.
62. Rosenblatt S, Irikura K, Caday DG, Finklestein SP, Moskowitz MA. Basic fibroblast growth factor (bFGF) dilates rat pial arterioles. J Cereb Blood Flow Metab 14:70-74, 1994.

63. Cuevas P, Carceller F, Ortega S et al. Hypotensive activity of fibroblast growth factor. Science 254:1208-1210, 1991.

64. Fisher M, Meadows M-E, Do T et al. Delayed treatment with intravenous basic fibroblast growth factor reduces infarct size following permanent cerebral ischemia in rats. J Cereb Blood Flow Metab 15:953-959, 1995.

65. Jiang N, Finklestein SP, Do T, Caday CG, Charette M, Chopp M. Delayed intravenous administration of basic fibroblast growth factor (bFGF) reduced infarction volume in a model of focal cerebral ischemia/reperfusion in the rat. $J$ Neurol Sci 139:173-179, 1996.

66. Reynolds BA, Weiss S. Generation of neurons and astrocytes from isolated cells of the adult mammalian central nervous system. Science 255:1707-1710, 1992.

67. Gould E, Reeves AJ, Graziano MSA, Gross CG. Neurogenesis in the neocortex of adult primates. Science 286:548-552, 1999.

68. Takagi Y, Nozaki K, Takahashi J, Ishikawa M, Hashimoto N. Proliferation of neuronal precursor cells in the dentate gyrus is accelerated after transient forebrain ischemia in mice. Brain Res 831:283-287, 1999.

69. Nakatomi H, Kuriu T, Okabe S, Yamamoto S, Hantano O, Kawahara $\mathrm{N}$ et al. Regeneration of hippocampal pyramidal neurons after ischemic brain injury by recruitment of endogenous neural progenitors. Cell 110:429-441, 2002.

70. Li Y, Chen J, Chopp M. Cell proliferation and differentiation from ependymal, subependymal and choroid plexus cells in response to stroke in rats. J Neurol Sci 193:137-146, 2002.

71. Modo M, Stroemer P, Tang E, Patel S, Hodges H. Effects of implantation site of stem cell grafts on behavioral recovery from stroke damage. Stroke 33:2270-2278, 2002.

72. Woodbury D, Schwarz EJ, Prockop DJ, Black IB. Adult rat and human bone marrow stromal cells differentiate into neurons. J Neurosci Res 61:364-370, 2000.

73. Li Y, Chen J, Chen XG, Wang L, Gautam SC, Xu YX et al. Human marrow stromal cell therapy for stroke in rat: neurotrophins and functional recovery. Neurology 59:514-523, 2002.

74. Kondziolka D, Wechsler L, Goldstein S, Meltzer C, Thulborn KR, Gebel J et al. Transplantation of cultured human neuronal cells for patients with stroke. Neurology 55:565-569, 2000.

75. The Hypothermia after Cardiac Arrest Study Group. Mild therapeutic hypothermia to improve the neurologic outcome after cardiac arrest. $N$ Engl J Med 346:549-556, 2002.

76. Wang Y, Lim L, Levi C, Heller R, Fisher J. Influence of admission body temperature on stroke mortality. Stroke 31:404-409, 2000.

77. Krieger D, De Georgia M, Abou-Chebl, A Andrefsky JC, Sila CA, Katzan IL et al. Cooling for acute ischemic brain damage (COOL AID). Stroke 32:1847-1854, 2001.

78. Georgiadis D, Schwarz S, Kollmar R, Schwab S. Endovascular cooling for moderate hypothermia in patients with acute stroke: first results of a novel approach. Stroke 32:2550-2553, 2001.

79. Dewar D, Yam P, McCulloch J. Drug development for stroke: importance of protecting cerebral white matter. Eur J Pharmacol 375:47-50, 1999.

80. Hunter AJ, Mackay KB, Roger DC. To what extent have functional studies of ischemia in animals been useful in the assessment of potential neuroprotective agents? Trends Pharmacol Sci 19:5966, 1998.

81. Duncan PW, Jorgensen HS, Wade DT. Outcome measures in acute stroke trials: a systematic review and some recommendations to improve practice. Stroke 31:1429-1438, 2000.

82. Demchuk AM, Buchan AM. Predictors of stroke outcome. Neurol Clin 18:455-473, 2001.

83. Grotta JC. Acute stroke therapy at the millennium: consummating the marriage between the laboratory and bedside: the Feinberg lecture. Stroke 30:1722-1728, 1999.

84. Zivin JA. Factors determining the therapeutic window for stroke Neurology 50:599-603, 1998.

85. Du C, Hu R, Csernansky CA, Hsu CY, Choi DW. Very delayed infarction after mild focal cerebral ischemia: a role for apoptosis? J Cereb Blood Flow Metab 16:195-201, 1996. 
86. Jonas A, Aiyagari V, Vieira D, Figueroa M. The failure of neuronal protective agents versus the success of thromolysis for the treatment of ischemic stroke: the predictive value of animal models. Ann NY Acad Sci 939:257-267, 2001.

87. Dyker AG, Lees KR. Duration of neuroprotective treatment for ischemic stroke. Stroke 29:535-542, 1998.

88. Malakoff D. Bayes offers a "new" way to make sense of numbers. Science 286:1460-1464, 1999.

89. Wilson LJT, Hareendran A, Grant M, Baird T, Schulz UG, Muir $\mathrm{KW}$ et al. Improving the assessment of outcomes in stroke: use of a structured interview to assign grades on the modified ranking scale. Stroke 33:2243-2246, 2002.

90. Tilley BC, Marler J, Geller NL, Lu M, Legler J, Brott T et al. Use of a global test for multiple outcomes in stroke trials with application to the National Institute of Neurological Disorders and Stroke t-PA Trial. Stroke 27:2136-2142, 1996

91. Zivin JA, Mazzarella V. Tissue plasminogen activator plus glutamate antagonist improves outcome after embolic stroke. Arch Neurol 48:1235-1238, 1991 .

92. Bowes MP, Rothlein R, Fagan SC, Zivin JA. Monoclonal antibodies preventing leukocyte activation reduce experimental neurologic injury and enhance efficacy of thrombolytic therapy. Neurology 45:815-819, 1995.

93. Lyden PD, Lonzo L, Nunez S. Combination chemotherapy extends the therapeutic window to 60 minutes after stroke. J Neurotrauma 12:223-230, 1995. 